Artículo de investigación

\title{
La satisfacción laboral como efecto moderador entre las prácticas organizacionales de recursos humanos y las líneas estratégicas de responsabilidad social corporativa en México
}

\author{
Sergio Madero-Gómez * \\ Profesor Titular, Departamento de Gestión y Liderazgo, Tecnológico de Monterrey, Monterrey, México. \\ smadero@tec.mx
}

\section{Resumen}

El objetivo del presente artículo fue conocer el efecto moderador que tiene la satisfacción laboral en las prácticas de recursos humanos y en las líneas estratégicas de la responsabilidad social empresarial, a saber, ética empresarial, desarrollo sustentable, desarrollo social y calidad de vida. Para esto se empleó una metodología de tipo cuantitativa que implicó el diseño de un cuestionario con 37 reactivos, a partir del cual se recolectaron 458 datos de personas que trabajan en empresas del Noreste de México. Los principales hallazgos destacan que la práctica de recursos humanos que mayor relevancia tiene es cumplir a tiempo con las expectativas de los colaboradores; además, se observa que la satisfacción laboral tiene un efecto moderador en las variables propuestas. Se concluye que estas prácticas son clave en la implementación de las estrategias de responsabilidad social.

Palabras clave: prácticas de recursos humanos; responsabilidad social empresarial; México.

\section{Job satisfaction as a moderating effect between organizational practices of human resources and the strategic lines of corporate social responsibility in México}

\begin{abstract}
The aim of this paper was to know the moderating effect of job satisfaction in human resource practices and in strategic lines of corporate social responsibility, as business ethics, sustainable development, social development, and quality of life. For this, a quantitative methodology that implied the design of a questionnaire with 37 items was applied, collecting a sample of 458 data from people who work in companies in Northeast Mexico. The main findings show that the most relevant human resource practice is to meet the expectations of employees on time. Furthermore, it is observed that job satisfaction has a moderating effect on the proposed variables. It is concluded that these practices are key to the implementation of social responsibility strategies.
\end{abstract}

Keywords: human resources practices; corporate social responsibility; Mexico.

\section{A satisfação no trabalho como efeito moderador entre as práticas organizacionais de recursos humanos e as linhas estratégicas de responsabilidade social corporativa no México}

\section{Resumo}

O objetivo deste artigo foi conhecer o efeito moderador que a satisfação no trabalho tem nas práticas de recursos humanos e nas linhas estratégicas da responsabilidade social empresarial, isto é a ética empresarial, o desenvolvimento sustentável, o desenvolvimento social e a qualidade de vida. Para isso, utilizou-se uma metodologia quantitativa que implicou na elaboração de um questionário com 37 itens, do qual foram coletados 458 dados de pessoas que trabalham em empresas no Nordeste do México. As principais conclusões destacam que a prática de recursos humanos mais relevante é atender às expectativas dos funcionários no prazo; além disso, observase que a satisfação no trabalho tem efeito moderador sobre as variáveis propostas. Conclui-se que essas práticas são fundamentais na implementação de estratégias de responsabilidade social.

Palavras-chave: práticas de recursos humanos; Responsabilidade Social Empresarial; México.

\section{* Autor para dirigir correspondencia}

Clasificación JEL: M12; M14; M50.

Cómo citar: Madero-Gómez, S. (2020). La satisfacción laboral como efecto moderador entre las prácticas organizacionales de recursos humanos y las líneas estratégicas de responsabilidad social corporativa en México. Estudios Gerenciales, 36(157), 391-401. https://doi.org/10.18046/j.estger.2020.157.3790

DOI: https://doi.org/10.18046/j.estger.2020.157.3790

Recibido: 11-nov-2019

Aceptado: 02-sep-2020

Publicado: 30-dic-2020 


\section{Introducción}

En la actualidad, las empresas tienden a adoptar procesos innovadores de transformación e intentan enfocarse en trabajar cada vez más en función de la responsabilidad social empresarial (RSE); pero su implementación exitosa implica un cambio en las prácticas de gestión, primero en los niveles altos de la organización, pues se requiere convencimiento y aceptación para poder lograr el cambio y, posteriormente, involucrar a los diversos grupos de interés (Abad-Segura, Cortés-García y Belmonte-Ureña, 2019; Rojas-Vargas y Madero-Gómez, 2018; Becker y Smidt, 2016; Arnaud y Wasieleski, 2014; Michelini y Fiorentino, 2012).

Hiswais, Wulff, Vidman y Macassa (2020) y Macassa, Francisco y McGrath (2017) mencionan que el nuevo contexto de los negocios, sobre todo en países emergentes con la influencia de compañías multinacionales es sus territorios, ha comenzado a poner en práctica una variedad de iniciativas y estrategias en beneficio de la sociedad y de su fuerza laboral, como programas para el mejoramiento de la salud y estrategias para disminuir los riesgos psicosociales en el trabajo (Uribe, Gutiérrez y Amézquita, 2020; Luna-Chávez, Anaya-Velasco y RamírezLira, 2019; Rubio-Avila y Gómez-Sánchez, 2018). Estos proyectos propenden a mejorar las condiciones de trabajo y se espera que se vean reflejados en la productividad y en la satisfacción de los trabajadores (López, Ojeda y Ríos, 2017; Voegtlin y Greenwood, 2016; Baumann-Pauly, Wickert, Spence y Scherer, 2013), así como en la calidad de su trabajo (Martínez-García, Sorribes y Celma, 2018). De esta forma, se transmite la idea de que las iniciativas realizadas deben tener un interés compartido entre ambas partes, es decir, la empresa y el colaborador, y deben actuar de manera inmediata tratando de mejorar el desempeño social, mediante las actividades propias de la función de recursos humanos.

Es necesario mencionar que existen diferentes razones que hacen a una empresa interesarse y poner en práctica las estrategias de RSE (Sardana, Gupta, Kumar y Terziovski, 2020; Yang, Colvin y Wong, 2013); por ejemplo, la motivación del consejo directivo, la propia convicción del dueño o de algún directivo y el liderazgo de los gerentes dentro y fuera de la empresa (Shashi, Cerchione, Singh y Dahiya, 2020; Bolton, Kim y O'Gorman, 2011). El interés de la empresa en estas estrategias también se debe a la presión ejercida por algún grupo de interés, originado por una renovación en su imagen o por la interpretación de métricas financieras, operativas y de desempeño (Barboza, Pede y Madero, 2019; Alshehhi, Nobanee y Khare, 2018; Elizondo y Madero, 2018; McWilliams y Siegel, 2000).

Para la implementación y el establecimiento de diversas prácticas de RSE, existe una serie de términos y conceptos que están poco definidos o que no se conocen (Voegtlin y Greenwood, 2016; Aguinis y Glavas, 2012); por lo tanto, los trabajadores tienden a malinterpretar las acciones emprendidas por la empresa, quizá por el desconocimiento de las implicaciones que estas tienen. De los enunciados anteriores se derivan entonces las siguientes preguntas de investigación: ¿por qué se le da importancia solo a ciertas estrategias de la responsabilidad social y no a todas en su conjunto?, ¿de qué manera las prácticas de recursos humanos influyen en la implementación y seguimiento de diversas estrategias de responsabilidad social en las empresas?, ¿cuál es el efecto que tiene la satisfacción laboral sobre las prácticas de recursos humanos y las líneas estratégicas de la responsabilidad empresarial?

Debido a que no existe suficiente evidencia en la literatura respecto a la manera en que se relacionan las prácticas organizacionales de recursos humanos con las líneas estratégicas de la RSE, en el entorno laboral mexicano, se ha considerado oportuno realizar la presente investigación, en la que se definen como objetivos conocer la percepción que las personas tienen sobre la importancia de las diversas prácticas organizacionales de recursos humanos que se realizan en las empresas en las que laboran y sobre las líneas estratégicas de la RSE (calidad de vida, desarrollo social, desarrollo sustentable y ética empresarial); identificar el efecto que estas tienen en las prácticas organizacionales de recursos humanos; $y$, finalmente, conocer el efecto moderador que tiene la satisfacción laboral entre las prácticas de recursos humanos en las empresas y las líneas estratégicas de la RSE.

El documento está compuesto por cuatro secciones más. La segunda es el marco teórico, en la que se exponen los diversos aspectos conceptuales relacionados con la investigación. Enseguida se muestra el proceso metodológico empleado, en el que se señala el modelo de la investigación, que es de tipo cuantitativa y descriptiva con un diseño no experimental y un tipo de muestreo no probabilístico. Luego, se exponen los resultados obtenidos $y$, finalmente, se presentan las conclusiones.

\section{Marco teórico}

En este apartado se hace una revisión de los diversos temas involucrados en la investigación; primero de los temas de RSE y de las cuatro líneas estratégicas que se consideran en el desarrollo del proyecto, luego se analiza la satisfacción en el trabajo y, por último, las prácticas organizacionales de recursos humanos.

\subsection{Responsabilidad social empresarial}

Una ESR es aquella que tiene el compromiso consciente y congruente de cumplir de manera íntegra con los planes y proyectos de la empresa; además la ayuda a cumplir sus objetivos tanto desde la perspectiva interna como desde la externa. Así lo exponen Barrena-Martínez, López- Fernández y Romero-Fernández (2019) y Peloza y Shang (2011), quienes consideran que se deben diseñar estrategias que se enfoquen en cumplir las expectativas mediante actividades en las que todos los grupos de interés estén involucrados, y de esta manera demostrar el respeto por los valores propios de la organización, los 
principios éticos, las personas, las comunidades, el medio ambiente y la construcción del bien común (Jamali, El Dirani y Harwood, 2015; Skudiene y Auruskeviciene, 2012; Freeman y Hasnaoui, 2011).

Por su parte, Mondy (2010) menciona que "la RSE es la obligación de los administradores al actuar en su capacidad oficial de servir o proteger los intereses de grupos distintos de ellos mismos" (p. 39), mientras que para Bohlander, Snell y Morris (2018) es "actuar a favor de los mejores intereses de las personas y las comunidades afectadas por sus actividades" (p. 11). Cabe mencionar que las prácticas empresariales se han estudiado desde diversas perspectivas: desde sus estructuras institucionales, sus sistemas internos, sus mecanismos de implementación y sus esquemas de gobernanza (Elston, 2019; Taneja, Taneja y Gupta, 2011). Estas han logrado un alcance mundial, a partir de las experiencias compartidas y de sus diversas formas de aplicación, para tratar de ajustarse a cada región de acuerdo con el mercado meta, pues cada uno tiene sus propias costumbres, tradiciones y prioridades (Arminen, Puumalainen, Pätäri y Fellnhofer, 2018; Celma, MartínezGarcía y Coenders, 2014; Sotome y Takahashi, 2014; Madueño, Jorge y Martínez-Martínez, 2013; Sanchez-Runde, Nardon y Steers, 2013; Madero y Navarro, 2010; Samy, Odemilin y Bampton, 2010; Tsoi, 2010; Torres-Baumgarten y Yucetepe, 2009). Otro aspecto que es relevante mencionar es que, mediante el diseño y el seguimiento de un código de prácticas empresariales (Consejo Coordinador Empresarial [CCE], 2010), una ESR promueve e impulsa el comportamiento ético y la cultura de competitividad responsable, que busca las metas y el éxito del negocio y contribuye al mismo tiempo al bienestar de la sociedad.

A continuación, se analizan las diversas dimensiones estratégicas que existen en la RSE. De acuerdo con Cajiga (s. f.), del Centro Mexicano de la Filantropía (CEMEFI), el distintivo ESR es un reconocimiento que se otorga en Latinoamérica y es un elemento estratégico de promoción que obtienen las empresas por asumir voluntaria y públicamente el compromiso de una gestión socialmente responsable, como parte de su cultura y estrategia de negocio, al haber sustentado el cumplimiento de los estándares propuestos en los siguientes indicadores, llamados también líneas estratégicas. Rosolen y Maclennan (2016) los definen como las dimensiones de la RSE: 1) pensamiento ético, relacionado con los aspectos de ética empresarial; 2l desarrollo sustentable o calidad ambiental; 3) vinculación con la sociedad o desarrollo social; y 4) calidad de vida de los trabajadores.

\subsubsection{Pensamiento ético}

En lo que corresponde a la primera línea estratégica, se puede mencionar que todas las empresas desde su creación tienen una obligación ética con todos los involucrados o grupos de interés, de acuerdo con el rol o función que tengan, y, a su vez, cada uno de los involucrados debe dar la importancia requerida a las diversas actividades relacionadas con este tema, tal como lo mencionan GodosDíez, Fernández-Gago y Martínez-Campillo (2011). De hecho, cada uno debe enfocar sus estrategias para mostrar un liderazgo responsable (Bondy, Moon y Matten, 2012; Gond, Igalens, Swaen y El Akremi, 2011), que alcance y trascienda en cada uno de los diferentes grupos de interés: propietarios, accionistas, empleados, clientes, proveedores y la comunidad en general - como lo señalan Lamberti y Lettieri (2009) - , no solo en términos de normas y deberes que se tienen que cumplir, sino en términos de principios y valores, como la libertad, la igualdad, la solidaridad, el respeto y el diálogo cordial entre todos (Dale, 2012; Davis y MacDonald, 2010; Lai-Chen y Ahmad, 2010).

Tomando en cuenta lo anterior, es conveniente señalar que el área de recursos humanos, dentro de las actividades que realiza para el personal, debe mantener una estrecha comunicación con la dirección general, ambas áreas consideradas clave dentro de la empresa. Por lo tanto, es necesario proponer la generación de elementos éticos fundamentales que permitan el desarrollo, la divulgación y el cumplimiento de los valores empresariales en las personas que ahí trabajan, como lo mencionan Greenwood (2013), Inyang, Awa y Enuoh (2011) y Davis y MacDonald (2010). Es decir, se debe buscar la construcción, conformación, seguimiento y consolidación de una empresa con una filosofía ética basada en al una cultura corporativa sólida (valores definidos y construidos colectivamente); b) el talento humano como capital principal de la empresa; c) la excelencia como una forma de trabajo; d) la transformación e innovación como aspiración fundamental; el la búsqueda de bienes, tanto tangibles como intangibles larmonía, cooperación, ausencia de conflictos, cordialidad, etc.); f) la atención de las necesidades de los colaboradores; y gl el cumplimiento oportuno de los acuerdos con los clientes y proveedores. Todo lo anterior en el marco de sus actuaciones y no solo para que sus accionistas puedan lograr los objetivos de rentabilidad, sino que puedan asumir la responsabilidad social por el valor de las acciones de la empresa, para ejercer así una dirección basada en los valores y en la que predomine un contrato moral de la empresa con sus integrantes, más allá de la existencia de un contrato legal o de la estructura de un gobierno corporativo para la repartición de utilidades (Guerci, Radaelli, Siletti, Cirella y Shani, 2015; Manroop, Singh y Ezzedeen, 2014; Podnar y Golob, 2007).

\subsubsection{Desarrollo sustentable}

En lo que respecta a la segunda línea estratégica, relacionada con el desarrollo sustentable, actualmente existe mucha preocupación por el mejor aprovechamiento de todos los recursos, principalmente medioambientales, así como la necesidad de tener mayor igualdad social y buscar un crecimiento económico sostenible y global (Organización de las Naciones Unidas [ONU], 2020; Elizondo y Madero, 2018). Con lo anterior, se espera generar una gran variedad de prácticas empresariales para aplicar estas iniciativas, tal como lo indican Henriques y Sadorsky (2006), quienes hacen 
referencia a las acciones de sustentabilidad realizadas en los países con economías emergentes.

Leidner, Baden y Ashleigh (2019), Haberberg, Gander, Rieple, Helm y Martin-Castilla (2010) y Van Marrewijk (2003) mencionan que la protección del medioambiente y el desarrollo sustentable son dos factores clave de una ESR; factores que deben estar alineados con las estrategias de la empresa y con las directrices de la función de recursos humanos, con la finalidad de ser generadoras de un cambio en el comportamiento y en las actitudes de los colaboradores. En las últimas décadas, la preocupación por la preservación del medioambiente y el desarrollo sostenible -que no implique la afectación de los recursos naturales, sobre todo de los que están alrededor de la empresaha adquirido una dimensión cada vez mayor y es materia relevante para establecer acuerdos y alianzas con todos los que trabajan en ello, por lo que las empresas pueden hacer mucho al respecto, en este caso con sus colaboradores y grupos de interés, aun con la ausencia de normas, leyes y políticas medioambientales emitidas por el sector gubernamental.

\subsubsection{Vinculación con la sociedad}

La siguiente línea estratégica está enfocada en la cercanía que tiene o debe tener una ESR con la sociedad, es decir, mantener una vinculación con la comunidad a partir de la misión del negocio - también llamada desarrollo social y que es un aspecto clave-. También se espera que las organizaciones realicen acciones que propicien el desarrollo de la sociedad, como parte de los planes y retos que se tienen (ONU, 2020; Elizondo y Madero, 2018), y que trabajen de manera más proactiva en conjunto con sus colaboradores y grupos de interés para atender diversas causas de índole social, como programas de voluntariado estratégico y no estratégico Gyves y O'Higgins (2008). Se espera, además, que busquen el bienestar público, más allá de la mera filantropía, debido a que en ocasiones no hay un punto de enlace con la misión y visión de la empresa, aunque de alguna manera, por el prestigio de la marca o por las características que tiene como empleador, se tienen resultados positivos que aportan a los indicadores deseados por el consejo directivo de la empresa.

Actualmente, en muchas empresas, las actividades de la función de recursos humanos se están enfocando, sobre todo, en la implementación de estrategias para atender los aspectos del comportamiento, tanto en la perspectiva humana como en la organizacional. Esto con el fin de generar un cambio y lograr sensibilizar a las personas sobre su entorno, es decir, ofrecer especial atención a su desarrollo como individuos y buscar principalmente su realización mediante las actividades que llevan a cabo en su lugar de trabajo, con el fin de generar mayor satisfacción por lo que hacen y que cada uno desarrolle de manera constante sus habilidades y capacidades. Tal desarrollo personal debe ser puesto al servicio de los demás, para apoyar y facilitar la implementación de las estrategias e iniciativas de desarrollo social que la empresa va planteando (Buciuniene y Kazlauskaite, 2012; Roos, Fernstrom y Pike, 2004).

\subsubsection{Calidad de vida de los trabajadores}

Otra de las líneas estratégicas que deben considerar las ESR es contribuir a mejorar la calidad de vida del trabajador dentro y fuera de las instalaciones de la empresa, mediante propuestas generadas en el área de recursos humanos, como programas de bienestar integral de las personas, fomento de la salud, planes de nutrición, salud mental y acondicionamiento físico. Así mismo, es importante considerar el diseño de las estrategias para la retribución tanto monetaria como no monetaria a los colaboradores, que van desde el salario emocional, el pago justo y equitativo, incluyendo esquemas de incentivos variables, hasta esquemas de desarrollo de talento (Madero, 2010; Vaiman, Scullion y Collings, 2012), sin considerar el origen, el género, el estado civil, la antigüedad y la experiencia que tenga la persona en el trabajo.

Por otra parte, es necesario analizar la manera en que las áreas de recursos humanos participan en el desarrollo de propuestas relevantes con el fin de mejorar las condiciones en el lugar de trabajo y el clima organizacional a través de directrices estratégicas, reforzadas por programas de bienestar integral, balance entre actividades personales y laborales y la comunicación eficiente en la que se destaque el trato digno a las personas. Estas acciones se deben realizar para favorecer y ayudar a los colaboradores a mantener la estabilidad laboral en la empresa; además, es importante considerar que en ambientes de incertidumbre o inestabilidad, las organizaciones deben enfocar sus esfuerzos en ofrecer esquemas que fomenten la estabilidad y la seguridad del empleo mediante el pago de los salarios justos, en tiempo y forma, y ofrecer las condiciones idóneas en el área de trabajo, para evitar accidentes y así disminuir los problemas ocasionados por la rotación y el ausentismo del personal (Celma et al., 2014).

\subsection{Satisfacción en el trabajo}

Otro componente importante en esta investigación es lo que Griffin, Phillips y Gully (2017) mencionan respecto a que "la satisfacción laboral es uno de los temas que más se estudian en el área del comportamiento organizacional y es una condición que refleja una serie de actitudes y sentimientos por parte de la persona hacia el trabajo que realiza" (p. 128). Por su parte, Robbins y Judge (2017) la definen como un sentimiento positivo respecto al puesto de trabajo, resultado de una evaluación de las características con las que se cuenta. Las principales causas de la satisfacción en el trabajo están influidas por las condiciones laborales, la variedad de las actividades que realiza la persona en su trabajo, la retroalimentación que recibe, el apoyo de la empresa, la interacción de los colegas y el trato recibido por parte de sus jefes o superiores. 
Robbins y Judge (2017) también mencionan que existen organizaciones que exigen a sus colaboradores contribuir de alguna manera a sus diversas iniciativas o estrategias sobre responsabilidad social, integrándolos a campañas de voluntariado, recolección de víveres para ayudar a la comunidad, servicios en comedores comunitarios, construcción de viviendas en la localidad en la que se encuentran, participación como facilitadores o instructores de clubes de lectura, entre otras. Estas actividades generan satisfacción a las personas que participan. La RSE es, por tanto, una tendencia positiva necesaria de responsabilidad y altruismo que puede contribuir de manera significativa en la satisfacción del personal y aporta diferentes beneficios para todos los grupos de interés.

Se observa que las personas que trabajan en organizaciones con evidencia de ser socialmente responsables, mediante prácticas de recursos humanos, muestran actitudes positivas y se sienten satisfechas con las actividades que realizan (Kim, Woo, Uysal y Kwon, 2018; Pérez, Fernández-Salinero y Topa, 2018; Collier y Esteban, 2007).

\subsection{Las prácticas organizacionales de recursos humanos}

En cuanto a las prácticas organizacionales relacionadas con la función de recursos humanos, se podrían considerar las que mencionan Barrena-Martínez et al. (2019), Voegtlin y Greenwood (2016), App, Merk y Büttgen (2012), Lis (2012) y Madero (2010, 2009), Lis (2012) y Madero $(2010,2009)$ respecto a la relevancia que tienen los factores de atracción y retención del talento, las oportunidades de promoción, de crecimiento y el desarrollo de habilidades, ya que son los aspectos más importantes que las personas consideran al momento de buscar empleo o al momento de aceptar una oferta formal de trabajo. Además de los aspectos anteriores, también están los sueldos y los salarios que ofrece la empresa, la capacitación y el entrenamiento; estas prácticas propias de la gestión de recursos humanos forman parte de la transferencia de conocimientos entre las personas para lograr la sustentabilidad de los procesos en la empresa, como lo indica Nicolopoulou (2011).

Las empresas deben hacer hincapié en el fortalecimiento de los procesos para la implementación exitosa de las prácticas de recursos humanos que, de alguna manera, influyen en la filosofía de la RSE, como lo son el cumplimiento de las expectativas de los colaboradores al integrarse en la organización y el acercamiento entre los trabajadores, sus familias y las empresas, para crear una ventaja competitiva en los procesos de atracción y retención del talento (Story, Castanheira y Hartig, 2016; App et al., 2012; Ehnert y Harry, 2012; Lis, 2012).

Por su parte, Calderón, Álvarez y Naranjo (2011) y Calderón, Naranjo y Álvarez (2010) mencionan la importancia de las prácticas de la gestión humana en los procesos de la RSE; destacan la relevancia que tienen en la construcción del capital social de la empresa, sobre todo por sus aportaciones en el mejoramiento de las actividades laborales. Por otra parte, Peláez-León, García y Azuero
(2014) y Jamali et al. (2015) destacan el aporte estratégico que tienen en el accionar diario de las empresas y en las personas, mientras que Leidner et al. (2019) lo hacen desde la perspectiva del impacto en el comportamiento de las personas como parte de las iniciativas propias de la función de recursos humanos, para concientizar sobre el cuidado del medioambiente.

Tomando en cuenta lo que Barrena-Martínez et al. (2019), Barrena-Martínez, López-Fernández y RomeroFernández (2017) y Alva (2011) mencionan respecto a que se tienen que crear las políticas y las condiciones para atraer talento a las organizaciones mediante una serie de prácticas organizacionales enfocadas a favorecer el ambiente de trabajo, para el desarrollo de la presente investigación se han considerado las siguientes prácticas de innovación y transformación de los procesos para fortalecer la RSE: 1) equidad de oportunidades, sin considerar el género ni la edad; 2) esquemas claros de comunicación; 3) sistema de quejas para mostrar la inconformidad del personal o sus sugerencias; 4 ) involucrar en la toma de decisiones al personal; 5) cumplir con lo que se promete en el proceso de contratación; 6) esquemas claros y justos en los procesos de la medición del desempeño; 7) oportunidad de promoción, crecimiento y desarrollo; (8) esquemas de compensaciones ligados al desempeño; 9) planes y programas de capacitación, así como el desarrollo de habilidades del personal de la empresa; 10) esquemas de reconocimiento; y 11) programas de acercamiento con las familias de los colaboradores. Es necesario señalar que se podrían incluir otras prácticas de gestión humana responsable, resultado de las iniciativas empresariales y de las adaptaciones a la situación actual, como prácticas de no discriminación, respeto a los derechos humanos, programas de inclusión, esquemas de salud y seguridad y voluntariado corporativo; pero por el momento se ha considerado analizar solo estas prácticas básicas de la función de recursos humanos.

\section{Metodología}

A continuación, se presentan las diversas actividades metodológicas realizadas a lo largo de la presente investigación. En la figura 1 se muestra el modelo de investigación propuesto, en el cual se puede observar la probable relación entre las diversas prácticas organizacionales y las cuatro líneas estratégicas o dimensiones de la responsabilidad social corporativa, mencionadas por Rosolen y Maclennan (2016). Se espera encontrar alguna influencia entre ellas y los efectos moderadores que tenga la satisfacción en el trabajo.

\subsection{Método de investigación}

El tipo de estudio realizado en la investigación cuantitativa es correlacional-explicativo, con un diseño ex post facto, no experimental, y un tipo de muestreo no probabilístico, a partir de una muestra por conveniencia. Solo se observaron las percepciones de los 458 entre- 
vistados, a través de los resultados de encuestas realizadas en su propio centro de trabajo, como lo menciona Hernández, Fernández y Baptista (2010), cuya jornada de trabajo es de 8 horas, es decir, trabajadores de tiempo completo de diversas empresas del sector manufacturero, ubicadas en la zona metropolitana de Monterrey, Nuevo León, México y que son identificadas como socialmente responsables. Cabe mencionar que, aunque se tenga un número aceptable de encuestas, los resultados obtenidos aplican solo para la muestra de participantes y no pueden ser generalizados a toda la población.

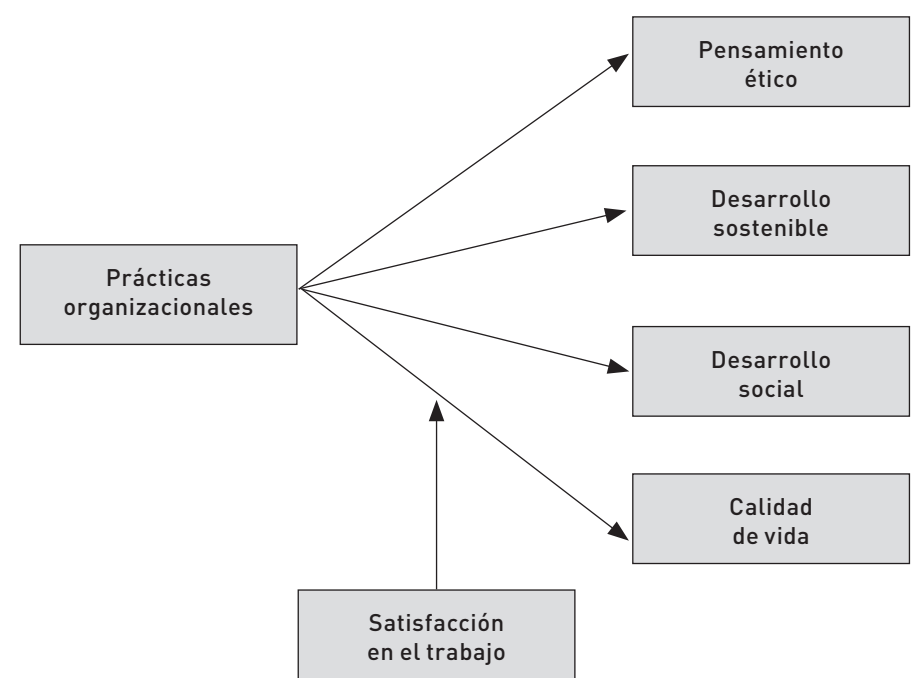

Figura 1. Modelo propuesto de la investigación

Fuente: elaboración propia.

Para el diseño y desarrollo del instrumento de medida, se consideraron las aportaciones realizadas en Alva (2011), Madero (2009) y Becerra (2006). La obtención de datos se llevó a cabo durante el semestre académico de eneromayo del 2016, con la aplicación de una encuesta que fue elaborada para este fin. La encuesta estuvo compuesta por 37 reactivos, de los cuales 31 son tipo Likert en escalas de 1 a 5 puntos, en la que 1 corresponde a estar totalmente en desacuerdo y 5 totalmente de acuerdo. Los reactivos se distribuyen de la siguiente manera: prácticas organizacionales (11 reactivos) (tabla 2), satisfacción en el trabajo (1 reactivo), pensamiento ético (4 reactivos), desarrollo sustentable (5 reactivos), desarrollo social (5 reactivos), calidad de vida (5 reactivos) y las variables demográficas ( 6 reactivos) en escalas nominales y ordinales (tabla 1). El sistema estadístico utilizado para el análisis de la información obtenida fue Statistical Package for the Social Science (SPSS) versión 24.0.

Respecto al análisis de los resultados, se realizó la prueba de validez y confiabilidad del instrumento con base en el trabajo de campo solo para las líneas estratégicas de la RSE, mediante un análisis factorial exploratorio (AFE); se obtuvieron cargas factoriales superiores a 0,40 en relación con la línea estratégica de calidad de vida; cuatro reactivos con indicadores estadísticamente válidos, lo que anula la afirmación "la empresa cuenta con políticas oficiales sobre estrés y bienestar integral"; mientras que para las otras tres variables de las líneas estratégicas todos los reactivos resultaron estadísticamente aceptables. Por tanto, se promediaron los resultados individuales de cada uno de los reactivos utilizados y se encontró para el pensamiento ético un alfa de Cronbach (a) de 0,835; para el desarrollo sustentable, de 0,900; mientras que, para el desarrollo social, de 0,922; y para la calidad de vida, de 0,754. En lo que respecta a las demás variables involucradas, no se realizó el AFE, debido a que no era necesaria su agrupación, pues las prácticas de recursos humanos se iban a utilizar de manera individual y para la satisfacción en el trabajo se utilizó solo un reactivo.

Enseguida se muestran las características de los participantes en la investigación, que sirven para conocer los datos de las personas involucradas. Se destaca que el 61,4\% es del género masculino; el $38,6 \%$, del género femenino, y el $66,0 \%$ tiene menos de 35 años (tabla 1).

Tabla 1. Características de los participantes

\begin{tabular}{lll}
\hline Participantes & $\mathrm{N}$ & Porcentaje \\
\hline Género & & \\
Masculino & 281 & 61,4 \\
$\quad$ Femenino & 177 & 38,6 \\
Estado civil & & \\
$\quad$ Soltero & 124 & 27,1 \\
Casado & 304 & 66,3 \\
Otro & 30 & 6,6 \\
Edad & & \\
Menos de 25 años & 14 & 3,1 \\
26- 35 años & 288 & 62,9 \\
Más de 35 años & 156 & 34,0 \\
Antigüedad en la empresa & & \\
Menos de un año & 29 & 6,3 \\
$\quad$ Entre 1 y 5 años & 173 & 37,8 \\
$\quad$ Entre 6 y 10 años & 166 & 36,2 \\
Más de 10 años & 90 & 19,7 \\
Antigüedad en el puesto & & \\
Menos de un año & 56 & 12,1 \\
$\quad$ Entre 1 y 5 años & 220 & 48,0 \\
$\quad$ Entre 6 y 10 años & 130 & 28,4 \\
Más de 10 años & 52 & 11,5 \\
Nivel académico & & \\
$\quad$ Maestría & 104 & 22,7 \\
$\quad$ Profesional & 297 & 64,9 \\
$\quad$ Preparatoria & 57 & 12,4 \\
& $\mathrm{~N}=458$ & $100 \%$ \\
\hline Fuente: & &
\end{tabular}

Fuente: elaboración propia.

\section{Resultados}

Uno de los objetivos de la investigación fue conocer la percepción de las personas acerca de las diversas prácticas organizacionales de recursos humanos que se tienen en las empresas en las que trabajan. A continuación, se muestran los promedios de cada una de las diversas acciones para hacer su análisis de manera individual y comprender la relevancia que tiene cada una de ellas en el entorno empresarial (tabla 2). 
Se puede observar que cumplir con lo que se promete en la contratación (CUMPL) ( $M D=3,89 ; D S=1,22$ ), realizar programas de acercamiento con las familias (ACERCA), $(M D=3,82 ; D S=1,11)$ y los programas de capacitación para el personal en todos los niveles organizacionales de la empresa (CAPAC) (MD=3,48; $D S=1,32)$ son las tres prácticas más relevantes de la función de recursos humanos que se deben desarrollar, con especial atención en la percepción de las personas que participaron en la investigación. Por su parte, los sistemas de quejas o sugerencias (SUGER) es la práctica que menor relevancia presenta $(M D=2,95 ; D S=1,36)$, lo cual señala que es un área de oportunidad para involucrar más a las personas o motivar más la participación entre los colaboradores.

En lo que respecta a la percepción acerca de las cuatro líneas estratégicas sociales de la responsabilidad, a continuación, se muestran los promedios de cada una de ellas, con el fin de analizarlas de manera individual y comprender la relevancia que tiene cada una en el entorno empresarial. Se observó que el desarrollo sustentable es la práctica que mayor relevancia tiene en la muestra participante $(M D=3,87 ; D S=0,99)$; las otras líneas estratégicas tienen otros promedios, que demuestran diversos niveles de importancia y diferentes prioridades en las personas, es decir, no todos en su conjunto tienen la misma relevancia, como se supuso en un comienzo (tabla 3 ).

Tabla 2. Prácticas organizacionales de recursos humanos

\begin{tabular}{|c|c|c|}
\hline & Promedio & $\begin{array}{l}\text { Desviación } \\
\text { estándar }\end{array}$ \\
\hline $\begin{array}{l}\text { a- Se ofrecen las mismas oportunidades a } \\
\text { todos los empleados (OPOR) }\end{array}$ & 3,23 & 1,21 \\
\hline b- Buena comunicación (COMUN) & 3,05 & 1,07 \\
\hline $\begin{array}{l}\text { c- Sistema de quejas, sugerencias o dudas } \\
\text { (SUGER) }\end{array}$ & 2,95 & 1,36 \\
\hline $\begin{array}{l}\text { d- Toma de decisiones que consideren a los } \\
\text { empleados (TD) }\end{array}$ & 3,03 & 1,26 \\
\hline $\begin{array}{l}\text { e- Se cumple lo que se promete en la } \\
\text { contratación (CUMPL) }\end{array}$ & 3,89 & 1,22 \\
\hline $\begin{array}{l}\text { f- Sistemas claros y justos de medición del } \\
\text { desempeño (JUST) }\end{array}$ & 3,03 & 1,21 \\
\hline g- Oportunidades de promoción (PROMO) & 3,05 & 1,27 \\
\hline $\begin{array}{l}\text { h- Compensaciones ligadas al desempeño } \\
\text { (COMP) }\end{array}$ & 3,33 & 1,18 \\
\hline $\begin{array}{l}\text { i- Programas de capacitación para todos } \\
\text { los niveles (CAPAC) }\end{array}$ & 3,48 & 1,32 \\
\hline $\begin{array}{l}\text { j- Esquemas de reconocimientos al } \\
\text { personal (RECON) }\end{array}$ & 3,23 & 1,12 \\
\hline $\begin{array}{l}\text { k- Programas de acercamiento con la } \\
\text { familia (ACERCA) }\end{array}$ & 3,82 & 1,11 \\
\hline
\end{tabular}

Fuente: elaboración propia.

Tabla 3. Líneas estratégicas de la RSE

\begin{tabular}{lll}
\hline & Promedio & Desviación estándar \\
\hline a- Pensamiento ético & 2,08 & 1,65 \\
b- Desarrollo sustentable & 3,87 & 0,99 \\
c- Vinculación con la sociedad & 3,53 & 1,01 \\
d- Calidad de vida de los trabajadores & 3,29 & 0,80 \\
\hline
\end{tabular}

Fuente: elaboración propia.
Con la finalidad de dar seguimiento a los objetivos planteados en la investigación, y para identificar el efecto que tienen las distintas líneas estratégicas de la RSE en las prácticas organizacionales de recursos humanos, se realizaron diversos modelos de regresión, utilizando como variables independientes las prácticas de recursos humanos y como variables dependientes las líneas estratégicas de la RSE; así mismo, en cada uno de los apartados se muestran los resultados obtenidos a partir de los análisis estadísticos para conocer el efecto moderador de la variable de satisfacción laboral. Se obtuvieron los resultados que se muestran a continuación.

\subsection{Pensamiento ético empresarial en función de las prácticas organizacionales de recursos humanos}

El modelo de regresión múltiple resultó estadísticamente significativo, $F(6,451)=45,26 ; p<0,001$, lo que explica en conjunto el $36,8 \%$ de la varianza $\left(R^{2}=0,368\right)$.

Se observó que los ítems predictores que más explican el pensamiento ético empresarial son las oportunidades de promoción (PROMO), administradas de forma transparente $(B=0,42, p<0,001)$, y los programas de acercamiento con las familias (ACERCA) $(B=0,39, p<0,001)$. También lo son el cumplimiento de las promesas en el proceso de la contratación (CUMPLE) $(B=0,10, p<0,05)$. Es importante señalar que las oportunidades de desarrollo (OPOR) $(B=-0,15$, $p<0,01)$, los procesos de comunicación (COMUN) $(B=-0,14$, $p<0,01)$ y el sistema de quejas y sugerencias (SUGER) $(B=-0,12$, $p<0,01)$ tienen efectos negativos en el pensamiento ético.

Al utilizar la variable de satisfacción en el trabajo como moderadora, se puede observar que el modelo de regresión para los trabajadores no satisfechos resultó estadísticamente significativo, $F(8,37)=63,12, p<0,001$; esto explica en conjunto el $91,7 \%$ de la varianza $\left(R^{2}=0,917\right)$, se integran en el modelo las compensaciones que tienen las personas (COMP) $(B=0,64, p<0,001)$ y los esquemas de reconocimiento formal en el trabajo (RECON) $(B=-0,59$, $p<0,001)$.

\subsection{Desarrollo sustentable en función de las prácticas organizacionales de recursos humanos}

En este apartado, el modelo de regresión múltiple resultó estadísticamente significativo, $F(6,451)=73,87$, $p<0,001$, lo que explica en conjunto el $48,9 \%$ de la varianza $\left(R^{2}=0,489\right)$.

Se pudo observar que los ítems predictores que más explican el desarrollo sustentable son el cumplimiento de las promesas en el proceso de contratación (CUMPL) $(B=0,48, p<0,001)$, además de los programas de acercamiento con las familias (ACERCA) $(B=0,40, p<0,001)$ y los esquemas de reconocimientos al personal (RECON) $(B=0,09, p<0,05)$. También es importante mencionar que las oportunidades de promoción (OPOR) $(B=-0,17, p<0,001)$, la buena comunicación (COMUN) $(B=-0,12, p<0,01)$ y el sistema de quejas o sugerencias (SUGER) $(B=-0,10, p<0,05)$ son variables predictoras, pero de manera negativa. 
Al utilizar la variable de satisfacción en el trabajo como moderadora, se puede observar que el modelo de regresión para los trabajadores no satisfechos resultó estadísticamente significativo, $F(3,42)=51,52, p<0,001$, lo que explica en conjunto el $77,7 \%$ de la varianza $\left(R^{2}=0,771\right)$, y la única variable independiente que se mantiene en los tres modelos realizados es el programa de acercamiento de las familias (ACERCA) $(B=0,82, p<0,001)$.

\subsection{Desarrollo social en función de las prácticas organi- zacionales de recursos humanos}

En este apartado, el modelo de regresión múltiple resultó estadísticamente significativo, $F(4,453)=58,64$, $p<0,001$, lo que explica en conjunto el $33,5 \%$ de la varianza $\left(R^{2}=0,335\right)$.

Los ítems predictores que más explican el desarrollo social son los programas de reconocimientos (RECON) $(B=0,42, p<0,001)$, la toma de decisiones que considera la opinión de los empleados (TD) $(B=0,23, p<0,001)$ y los programas de acercamiento con las familias (ACERCA) $(B=0,15, p<0,001)$, mientras que el sistema de quejas 0 sugerencias (SUGER) tiene un efecto inverso $(B=-0,24$, $p<0,001)$.

Al utilizar la variable de satisfacción en el trabajo como moderadora, se puede observar que el modelo de regresión para los trabajadores satisfechos resultó estadísticamente significativo, $F(4,237)=28,29, p<0,001$, lo que explica en conjunto el $31,2 \%$ de la varianza $\left(R^{2}=0,312\right)$. Se conservan en el modelo tres de las variables independientes: los programas de reconocimientos (RECON) $(B=0,35, p<0,001)$, la toma de decisiones que considera la opinión de los empleados (TD) $(B=0,54, p<0,001)$ y el sistema de quejas o sugerencias (SUGER), con un efecto inverso $(B=-0,43, \quad p<0,001)$; además, se integra como variable predictora la igualdad de oportunidades para los trabajadores (OPOR) $(B=-0,15, p<0,001)$.

\subsection{Calidad de vida de los trabajadores en función de las prácticas organizacionales de recursos humanos}

El modelo de regresión múltiple resultó estadísticamente significativo, $F(9,448)=104,67, p<0,001$, lo que explica en conjunto el $67,1 \%$ de la varianza $\left(R^{2}=0,671\right)$.

Se observó que los ítems predictores que más explican la calidad de los trabajadores son los programas de reconocimiento al trabajo realizado (RECON) $(B=0,27$, $p<0,001)$ y cuando se cumplen las promesas en el proceso de la contratación (CUMPL) $(B=0,27, p<0,001)$. También fue significativo que las oportunidades de promoción (PROMO) sean administradas de forma transparente $(B=0,19$, $p<0,001)$, los esquemas de compensaciones (COMP) $(B=0,19$, $p<0,001)$, los programas de acercamiento con las familias (ACERCA) $(B=0,15, p<0,001)$ y la igualdad de oportunidades para los trabajadores (OPOR) $(B=0,13, p<0,01)$.

Al utilizar la variable de satisfacción en el trabajo como moderadora, se puede observar que en ambos modelos de regresión los dos ítems predictores que aparecen son para los trabajadores satisfechos: la toma de decisiones que tiene en cuenta la opinión de los empleados (TD) $(B=0,14, p<0,01)$ y los programas de reconocimiento al trabajo realizado (RECON) $(B=0,35, p<0,001)$, mientras que para los no satisfechos los coeficientes son los siguientes: la toma de decisiones que tiene en cuenta la opinión de los empleados (TD) $(B=-0,49, p<0,001)$ y los programas de reconocimiento al trabajo realizado (RECON) $(B=-0,30$, $p<0,001)$, la diferencia entre ambas variables es el signo con el que afectan la variable dependiente que, en este caso, es la calidad de vida.

\section{Conclusiones}

Con el desarrollo de esta investigación se puede llegar, a partir de lo aquí mencionado, a diferentes conclusiones y reflexiones, entre ellas que las diversas prácticas organizacionales relacionadas con la función de recursos humanos son un factor clave en los procesos de implementación de la estrategia de RSE, como los programas de acercamiento entre la familia del colaborador y la empresa. Con base en esa estrategia, se espera lograr mayor arraigo y compromiso. Por otra parte, la capacitación a las personas que se contratan y el cumplimiento de sus expectativas son las prácticas que las personas consideran más importantes. Cabe destacar que, en la actualidad, existen iniciativas para la función de recursos humanos que es necesario considerar, con el fin de que las iniciativas de la RSE se puedan fortalecer, como los programas de inclusión y no discriminación, equidad de género, voluntariados corporativos, formación continua y promociones responsables, retribución equitativa y justa, entre otras (BarrenaMartínez et al., 2019; López et al. 2017; Ahmad 2015; PeláezLeón et al., 2014; Ehnert y Harris, 2012).

Así mismo, se observa que la percepción que tienen los trabajadores sobre las líneas estratégicas de la responsabilidad social son muy diversas, pues se esperaba que los resultados fueran similares y no fue así. Por tanto, se sugiere a los dirigentes empresariales y a los responsables de recursos humanos redefinir algunas estrategias para lograr resultados favorables, sobre todo, en el pensamiento o comportamiento ético y en la calidad de vida que se ofrece al trabajador. Por esto, si se desea promover una cultura empresarial ética enfocada en el cumplimiento de aspectos de la RSE, se debe inspirar y motivar a los involucrados en el cumplimiento de la normativa ética de la empresa, y que pongan todas sus capacidades al servicio de los demás, con participación y propuestas para vincularse con la sociedad (Ferreira y Real de Olivera, 2014).

Al analizar las cuatro líneas estratégicas propuestas por le CEMEFI, Cajiga (s. f.) favoreció la reflexión para la comprensión de los objetivos de la investigación, pues se puede observar que actualmente las personas y las empresas le dan importancia a cada una de ellas, de acuerdo con sus propios intereses, es decir, se enfocan en sus prácticas de mejoramiento medioambiental con la implementación de estrategias sustentables para la 
prevención de emisiones contaminantes y programas de reciclado de aguas. Otras estrategias, implementadas por el sector industrial en el que se encuentran, le dan importancia al desarrollo social, por medio de compañas de recolección de bienes para compartir en la comunidad; otras más invitan a sus colaboradores a participar como voluntarios en acciones de mejora de escuelas y albergues o bien a aliarse con organizaciones de la sociedad civil.

En lo que respecta a la primera línea estratégica relacionada con el pensamiento ético o con la filosofía empresarial, las empresas cumplen como un trámite o como un requisito más en los procesos del gobierno corporativo, que se activa solo cuando llega a existir alguna situación de corrupción, falta de probidad, fraude o corrupción que requiere la decisión de un comité interno. Por último, el tema que más relevancia tiene en esta investigación es el relacionado con la calidad de vida en el trabajo, pues las personas son una parte muy importante en el proceso productivo de las empresas. Por consiguiente, se puede concluir que las empresas le deben dar mayor importancia a la calidad de vida para tener un beneficio común, que logre mejorar la satisfacción del trabajador, pues, de acuerdo con los resultados obtenidos, se observa que esta sí tiene un efecto moderador entre las prácticas organizacionales de recursos humanos y las líneas estratégicas de la RSE.

Una de las limitaciones de la investigación fue que las prácticas organizacionales de recursos humanos utilizadas pueden considerarse como básicas, pero se podrían ampliar o incluir algunas otras. Es importante mencionar que, debido a los cambios recientes ocasionados por la contingencia sanitaria por la COVID-19, la función de recursos será un factor determinante en la implementación de las mejores prácticas para las empresas. En lo que respecta a la elaboración de los análisis estadísticos de las variables involucradas en el modelo, sería conveniente incluir algunas sociodemográficas, como género, estado civil, edad y antigüedad en el puesto de trabajo, entre otras, y agregar en el diseño del cuestionario los datos de identificación de las empresas participantes. Otra limitación fue que los resultados del estudio son representativos para la región geográfica donde se realizó la investigación, pero no pueden ser generalizados para toda la población, por lo que se considera un área de oportunidad para diseñar investigaciones futuras y poder replicar el estudio en otras ciudades y en otros sectores industriales. En esas futuras investigaciones se podría identificar si las prácticas de recursos humanos presentan el mismo comportamiento con las líneas estratégicas de la RSE, pues en la literatura mexicana o latinoamericana no se han encontrado suficientes evidencias de estudios similares, motivo por el cual, para los autores, esta investigación tiene relevancia.

Con la reflexión anterior, se puede observar una línea de investigación para desarrollar en el futuro, comprender el impacto que tiene la situación ocasionada por la COVID-19 en cada una de las líneas estratégicas de la RSE y conocer su evolución en los diversos métricos de la empresa, como son rentabilidad, productividad, clima laboral, factores de riesgo social, ambiental y laboral.

Para terminar, el CEMEFI, por medio de Villalobos (2020), con referencia a la problemática ocasionada por la propagación global de la COVID-19, destaca que la responsabilidad social en situaciones de crisis e incertidumbre "es cuidar, cuidarse y apoyar a los más vulnerables" (p. 1) y hace una invitación para que "todas las fundaciones y organizaciones de la sociedad civil y a todas las empresas, especialmente las que son ESR, a que continúen actuando con responsabilidad y compromiso en un momento difícil para todos. Es tiempo de dar continuidad a las acciones que permitan preservar la vida de todos y contribuir al bienestar de las comunidades" (p. 1).

\section{Conflicto de intereses}

El autor declara no tener ningún conflicto de intereses.

\section{Bibliografía}

Abad-Segura, E., Cortés-García, F. J. y Belmonte-Ureña, L. J. (2019). The sustainable approach to corporate social responsibility: A global analysis and future trends. Sustainability, 11(19), 5382. https://doi.org/10.3390/su11195382

Aguinis, H. y Glavas, A. (2012). What we know and don't know about corporate social responsibility: A review and research agenda. Journal of Management, 38(4), 932-968. https://doi.org/10.1177/0149206311436079

Ahmad, S. (2015). Green human resource management: Policies and practices. Cogent Business and Management, 2(1), 1-13. https://doi.org/10.1080/23311975.2015.1030817

Alshehhi, A., Nobanee, H. y Khare, N. (2018). The impact of sustainability practices on corporate financial performance: Literature trends and future research potential. Sustainability, 10(2), 494, 1-25. https://doi.org/10.3390/su10020494

Alva, S. (2011). Empresa admirada: la receta. México D. F.: LID.

App, S., Merk, J. y Büttgen, M. (2012). Employer branding: Sustainable HRM as a competitive advantage in the market for high-quality employees. Management Revue, 23(3), 262-278. https://doi.org/10.1688/1861-9908_mrev_2012_03_App

Arminen, H., Puumalainen, K., Pätäri, S. y Fellnhofer, K. (2018). Corporate social performance: Inter-industry and international differences. Journal of Cleaner Production, 177, 426-437. https://doi.org/10.1016/j.jclepro.2017.12.250

Arnaud, S. y Wasieleski, D. (2014). Corporate humanistic responsibility: Social performance through managerial discretion of the HRM. Journal of Business Ethics, 120, 313-334. https://doi.org/10.1007/s10551-013-1652-z

Barboza, G., Pede, V. y Madero, S. (2019). Shared social responsibility. Dual role of consumers as stakeholders in firm strategy. Social Responsibility Journal, ahead-of-print. https://doi.org/10.1108/SRJ-07-2019-0244

Barrena-Martínez, J., López-Fernández, M. y Romero-Fernández, P. (2017). Socially responsible human resource policies and practices: Academic and professional validation. European Research on Management and Business Economics, 23(1), 55-61. https://doi.org/10.1016/j.iedeen.2016.05.001

Barrena-Martínez, J., López-Fernández, M. y Romero-Fernández, P. (2019). Towards a configuration of socially responsible human resource management policies and practices: Findings from an academic consensus, The International Journal of Human Resource Management, 30(17), 2544-2580. https://doi.org/10.1080/09585192.2017.1332669

Baumann-Pauly, D., Wickert, C., Spence, L. J. y Scherer, A. G. (2013). Organizing corporate social responsibility in small and large firms: Size matters. Journal of Business Ethics, 115(4), 693-705. https://doi.org/10.1007/s10551-013-1827-7 
Becerra, I. (2006). Correlación y efecto entra prácticas organizacionales y actitudes del trabajador mexicano en la industria de telecomunicaciones (tesis de posgrado). (MATI), Tecnológico de Monterrey, México.

Becker, K. y Smidt, M. (2016). A risk perspective on human resource management: A review and directions for future research. Human Resource Management Review, 26, 149-165.

https://doi.org/10.1016/j.hrmr.2015.12.001

Bohlander, G., Snell, S. y Morris, S. (2018). Administración de recursos humanos (17. a ed.) México. D. F.: Cengage Learning.

Bolton, S., Kim, R. y O'Gorman, K. (2011). Corporate responsibility as a dynamic internal organizational process: A case study. Journal of Business Ethics, 101, 61-74. https://doi.org/10.1007/s10551-010-0709-5

Bondy, K., Moon, J. y Matten, D. (2012). An institution of corporate social responsibility (CSR) in multi-national corporations (MNCs): Form and implications. Journal of Business Ethics, 111(2), 281-299. https://doi.org/10.1007/s10551-012-1208-7

Buciuniene, I. y Kazlauskaite, R. (2012). The linkage between HRM, CSR and performance outcomes. Baltic Journal of Management, 7(1), 5-24. https://doi.org/10.1108/17465261211195856

Calderón, G., Naranjo, J. C. y Álvarez. C. M. (2010). Gestión humana en la empresa colombiana: sus características, retos y aportes. Una aproximación a un sistema integral. Cuadernos de Administración, 23(4), 13-36.

Calderón, G., Álvarez, C. M. y Naranjo, J. C. (2011). Papel de gestión humana en el cumplimiento de la responsabilidad social empresarial. Estudios Gerenciales, 27(118), 163-188. https://doi.org/10.1016/S0123-5923(11)70151-6

Cajiga, J. F. (s. f.). El concepto de responsabilidad social empresarial. Centro Mexicano para la Filantropía (CEMEFI). Recuperado el 29 de marzo de 2020, de: https://www.cemefi.org/esr/images/stories/pdf/esr/concepto_esr.pdf

CCE (2010). Código de Mejores Prácticas Corporativas, México. Recuperado el 29 de marzo de 2020, de: http://cce.org.mx/sites/default/files/CodigoMejoresPracticas.pdf

Celma, D., Martínez-Garcia, E. y Coenders, G. (2014). Corporate social responsibility in human resource management: An analysis of common practices and their determinants in Spain. Corporate Social Responsibility and Environmental Management, 21(2), 82-99. https://doi.org/10.1002/csr.1301

Collier, J. y Esteban, R. (2007). Corporate social responsibility and employee commitment. Business Ethics A European Review, 16(1), 1933. https://doi.org/10.1111/j.1467-8608.2006.00466.x

Dale, K. (2012). The employee as 'Dish of the Day': The ethics of the consuming/consumed self in human resource management. Journal of Business Ethics, 111, 13-24. http://dx.doi.org/10.1007/s10551-012-1437-9

Davis, D. y MacDonald, J. B. (2010). Improving the promotion of CSR initiatives: A framework for understanding stakeholder communications from a dynamic learning perspective. Academy of Marketing Studies Journal, 14(2), 77-93.

Ehnert, I. y Harry, W. (2012). Recent developments and future prospects on sustainable human resource management: Introduction to the special issue. Management Revue, 23(3), 221-238. http://dx.doi.org/10.2307/41783719

Elizondo, N. y Madero, S. (2018). La globalización ante los retos de sustentabilidad, económico-financieros y organizacionales. En J., Gómez-Romero, J. Sánchez-Cano y F. Villarreal Solís (Eds.), La globalización ante los retos de la sustentabilidad, económico-financieros y organizacionales (pp. 233-253). Durango: Editorial UJED.

Elston, J. (2019). Corporate governance: What we know and what we don'n know. Journal of Industrial and Business Economics, 46, 147-156. https://doi.org/10.1007/s40812-019-00115-z

Ferreira, P. y Real de Oliveira, E. (2014). Does corporate social responsibility impact on employee engagement? Journal of Workplace Learning, 26(3/4), 232-248. https://doi.org/10.1108/JWL-09-2013-0070

Freeman, I. y Hasnaoui, A. (2011). The meaning of corporate social responsibility: The vision of four nations. Journal of Business Ethics, 100(3), 419-443. https://doi.org/10.1007/s10551-010-0688-6
Godos-Díez, J., Fernández-Gago, R. y Martínez-Campillo, A. (2011). How important are CEOs to CSR practices? An analysis of the mediating effect of the perceived role of ethics and social responsibility. Journal of Business Ethics, 98(4), 531-548.

https://doi.org/10.1007/s10551-010-0609-8

Gond, J., Igalens, J., Swaen, V. y El Akremi, A. (2011). The human resources contribution to responsible leadership: An exploration of the CSR-HR interface. Journal of Business Ethics, 98, 115-132. https://doi.org/10.1007/978-94-007-3995-6_10

Greenwood, M. (2013). Ethical analyses of HRM: A review and research agenda. Journal of Business Ethics, 114(2), 355-366. https://doi.org/10.1007/s10551-012-1354-y

Griffin, R., Phillips, J. y Gully, S. (2017). Comportamiento organizacional: Administración de personas y organizaciones (12. ${ }^{a}$ ed.). México. D. F: Cengage Learning.

Guerci, M., Radaelli, G., Siletti, E., Cirella, S. y Shani, R. (2015), The impact of human resource management practices and the corporate sustainability on organizational ethical climates: An employee prospective. Journal of Business Ethics, 126, 325-342. https://doi.org/10.1007/s10551-013-1946-1

Gyves, S. y O'Higgins, E. (2008). Corporate social responsibility: An avenue for sustainable benefit for society and the firm? Society and Business Review, 3(3), 207-223. https://doi.org/10.1108/17465680810907297

Haberberg, A., Gander, J., Rieple, A., Helm, C. y Martin-Castilla, J. (2010). Institutionalizing idealism: The adoption of CSR practices. Journal of Global Responsibility, 1(2), 366-381.

https://doi.org/10.1108/20412561011079443

Henriques, I. y Sadorsky, P. (2006). The adoption of environmental management practices in a transition economy. Comparative Economic Studies, 48, 641-661. https://doi.org/10.1057/palgrave.ces.8100185

Hernández, R., Fernández, C. y Baptista, M. (2010). Metodología de la

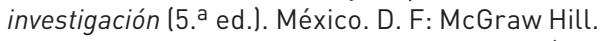

Hiswais, A., Wulff, C., Vidman, A. y Macassa, G. (2020). Corporate social responsibility and external stakeholders' health and wellbeing: $A$ viewpoint. Journal of Public Health Research, 9(1), 1742, 27-30. https://doi.org/10.4081/jphr.2020.1742

Inyang, B., Awa, H. y Enuoh, R. (2011). CSR-HRM Nexus: Defining the role engagement of the human resources professionals. International Journal of Business and Social Science, 2(5), 118-126.

Jamali, D. R., El Dirani, A. M. y Harwood, I. A. (2015). Exploring human resource management roles in corporate social responsibility: The CSR-HRM co-creation model. Business Ethics: A European Review, 24(2), 125-143. https://doi.org/10.1111/beer.12085

Kim, H., Woo, E., Uysal, M. y Kwon, N. (2018). The effects of corporate social responsibility (CSR) on employee well-being in the hospitality industry. International Journal of Contemporary Hospitality Management, 30(3), 1584-1600.

https://doi.org/10.1108/IJCHM-03-2016-0166

Lai-Chen, W. y Ahmad, J. (2010). Incorporating stakeholder approach in corporate social responsibility (CSR): A case study at multinational corporations (MNCs) in Penang. Social Responsibility Journal, 6(4), 593-610. https://doi.org/10.1108/17471111011083464

Lamberti, L. y Lettieri, E. (2009). CSR practices and corporate strategy: Evidence from a longitudinal case study. Journal of Business Ethics, 87(2), 153-168. https://doi.org/10.1007/s10551-008-9876-z

Leidner, S., Baden, D. y Ashleigh, M. (2019). Green (environmental) HRM: Aligning ideals with appropriate practices. Personnel Review, 48(5), 1169-1185. https://doi.org/10.1108/PR-12-2017-0382

Lis, B. (2012). The relevance of corporate social responsibility for a sustainable human resource management: An analysis of organizational attractiveness as a determinant in employees' selection of a (Potential) employer. Management Revue, 23(3), 279-295.

López, S. A., Ojeda, H. J. y Ríos, M. M. (2017). La responsabilidad social empresarial desde la percepción del capital humano. Estudio de Caso. Revista de Contabilidad-Spanish Accounting Review, 20(1), 36-46.

Luna-Chávez, E. A., Anaya-Velasco, A. y Ramírez-Lira, E. (2019). Diagnóstico de las percepciones de los factores de riesgo psicosociales en el trabajo del personal de una industria manufacturera. Estudos de Psicologia (Campinas), 36, 1-10. http://dx.doi.org/10.1590/1982-0275201936e180148 
Macassa, G. Francisco, J. y McGrath, C. (2017). Corporate social responsibility and population healt. Health Science Journal, 11(5), 1-6.

Madero, S. (2010). Factores relevantes del desarrollo profesional y de compensaciones en la carrera laboral del trabajador. Contaduría y Administración UNAM, 232,109-130.

Madero, S. (2009). Factores de atracción y retención del mercado laboral, para empleados potenciales. Economía, Gestión y Desarrollo, 7, 131-147.

Madero, S. y Navarro, M. (2010). Como se muestra la responsabilidad social en México a través de internet. Un análisis cualitativo. Esic Market, 135, 91-118.

Madueño, J. H., Jorge, M. L. y Martínez-Martínez, D. (2013). Relación entre responsabilidad social y performance en las pequeñas y medianas empresas: revisión bibliográfica. Cuadernos de Gestión, 13(2), 39-65. https://doi.org/10.5295/cdg.120360jh

Manroop, L., Singh, P. y Ezzedeen, S. (2014). Human resource systems and ethical climates: A resource-based perspective. Human Resource Management, 53(5), 795-816. https://doi.org/10.1002/hrm.21593

Martínez-García, E., Sorribes, J. y Celma, D. (2018). Sustainable development through CSR in human resource management practices: The effects of the economic crisis on job quality. Corporate Social Responsibility and Environmental Management, 25(4), 441456. https://doi.org/10.1002/csr.1471

McWilliams, A. y Siegel, D. (2000). Corporate responsibility and financial performance: Correlation or misspecification? Strategic Management Journal. 21(5), 603-609.

https://doi.org/10.1002/(SICI)1097-0266(200005)21:5<603::AIDSMJ101>3.0.C0;2-3

Michelini, L. y Fiorentino, D. (2012). New business models for creating shared value. Social Responsibility Journal, 8(4), 561-577. https://doi.org/10.1108/17471111211272129

Mondy, R. W. (2010). Administración de recursos humanos (11. a ed.). México. D. F.: Pearson Educación.

Nicolopoulou, K. (2011). Towards a theoretical framework for knowledge transfer in the field of CSR and sustainability. Equality, Diversity and Inclusion: An International Journal, 30(6), 524-538.

https://doi.org/10.1108/02610151111157738

ONU (2020). Objetivos de Desarrollo Sostenible. Recuperado el 15 de julio de 2020, de:

https://www.un.org/sustainabledevelopment/es/objetivos-dedesarrollo-sostenible/

Peláez-León, J., García, S. M. y Azuero, R. A (2014). La relación estratégica entre gestión humana y la responsabilidad social empresarial: avances de una explicación en un caso colombiano. Suma de Negocios, 5(11), 15-28.

Peloza, J. y Shang, J. (2011). How can corporate social responsibility activities create value for stakeholders? A systematic review. Academy of Marketing Science Journal, 39(1), 117-135. http://dx.doi.org/10.1007/s11747-010-0213-6

Pérez, S., Fernández-Salinero, S. y Topa, G. (2018). Sustainability in organizations: perceptions of corporate social responsibility and Spanish employee's attitudes and behaviors. Sustainability, 10, 1-15. https://doi.org/10.3390/su10103423

Podnar, K. y Golob, U. (2007). CSR expectations: The focus of corporate marketing. Corporate Communications: An International Journal, 12(4), 326-340. https://doi.org/10.1108/13563280710832498

Robbins, S. y Judge, T. (2017). Comportamiento organizacional (17. a ed.). México. D. F.: Pearson Educación.

Rojas-Vargas, N. y Madero-Gómez, S. (2018). La responsabilidad social corporativa: contexto histórico y relación con las teorías administrativas. Conciencia Tecnológica, 55(1), 29-38.

Roos, G., Fernstrom, L. y Pike, S. (2004). Human resource management and business performance measurement. Measuring Business Excellence, 8(1), 28-37. https://doi.org/10.1108/13683040410524711
Rosolen, T.y Maclennan, M. (2016). Strategic human resource management and corporate social responsibility: Evidence from emerging markets. Revista Electrónica de Negocios Internacionais, 11(2), 66-80. https://doi.org/10.18568/1980-4865.11266-80

Rubio-Avila, S. M. y Gómez-Sánchez, R. V. (2018). Factores psicosociales en el trabajo. Revista Colombiana de Salud Ocupacional, 8(2), e-5427. https://doi.org/10.18041/2322-634X/rcso.2.2018.5427

Samy, M., Odemilin, G. y Bampton, R. (2010). Corporate social responsibility: A strategy for sustainable business success. An analysis of 20 selected British companies. Corporate Governance, 10(2), 203-217. https://doi.org/10.1108/14720701011035710

Sanchez-Runde, C., Nardon, L. y Steers, R. M. (2013). The cultural roots of ethical conflicts in global business. Journal of Business Ethics, 116(4), 689-701. https://doi.org/10.1007/s10551-013-1815-y

Sardana, D., Gupta, N., Kumar, V. y Terziovski, M. (2020). CSR "sustainability" practices and firm performance in an emerging economy. Journal of Cleaner Production, 258, 1-10. https://doi.org/10.1016/j.jclepro.2020.120766

Shashi, R. S., Cerchione, R., Singh, R. y Dahiya, R. (2020). Effect of ethical leadership and corporate responsibility on firm performance: $A$ systematic review. Corporate Social Responsibility and Environmental Management. 27(2), 409-429. https://doi.org/10.1002/csr.1824

Skudiene, V. y Auruskeviciene, V. (2012). The contribution of corporate social responsibility to internal employee motivation. Baltic Journal of Management, 7(1), 49-67. https://doi.org/10.1108/17465261211197421

Sotome, R. y Takahashi, (2014). Does the Japanese employment system harm productivity performance? A perspective from DEA-based productivity and sustainable HRM. Asia-Pacific Journal of Business Administration, 6(3), 225-246. https://doi.org/10.1108/APJBA-02-2014-0031

Story, J., Castanheira, F. y Hartig, S. (2016). Corporate social responsibility and organizational attractiveness: Implications for talent management. Social Responsibility Journal, 12(3), 484-505. https://doi.org/10.1108/SRJ-07-2015-0095

Taneja, S. S., Taneja, P. K. y Gupta, R. K. (2011). Researches in corporate social responsibility: A review of shifting focus, paradigms, and methodologies. Journal of Business Ethics, 101(3), 343-364. https://doi.org/10.1007/s10551-010-0732-6

Torres-Baumgarten, G. y Yucetepe, V. (2009). Multinational firms' leadership role in corporate social responsibility in Latin America. Journal of Business Ethics, 85, 217-224. https://doi.org/10.1007/s10551-008-9940-8

Tsoi, J. (2010). Stakeholders' perceptions and future scenarios to improve corporate social responsibility in Hong Kong and Mainland China. Journal of Business Ethics, 91(3), 391-404. https://doi.org/10.1007/s10551-009-0091-3

Uribe, F., Gutiérrez, J. y Amézquita, J. (2020). Crítica a las propiedades psicométricas de una escala de medición de factores de riesgo psicosocial propuesta en la NOM 035 de la STPS en México. Contaduría y Administración, 65(1), 1-32.

Vaiman, V., Scullion, H. y Collings, D. (2012). Talent management decision making. Management Decision, 50(5), 925-941. https://doi.org/10.1108/00251741211227663

Van Marrewijk, M. (2003). Concepts and definitions of CSR and corporate sustainability: Between agency and communion. Journal of Business Ethics, 44(2), 95-105. https://doi.org/10.1023/A:1023331212247

Villalobos, J. (2020). Mensaje del Presidente Ejecutivo del Cemefi, sobre la responsabilidad social frente al Covid-19. Recuperado el 31 de marzo de 2020 de:

https://www.cemefi.org/servicios/noticias/filantropicas/5997mensaje-del-presidente-ejecutivo-del-cemefi

Voegtlin, C. y Greenwood, M. (2016). Corporate social responsibility and human resource management: A systematic review and conceptual analysis. Human Resource Management Review, 26, 181-197. https://doi.org/10.1016/j.hrmr.2015.12.003

Yang, N., Colvin, C. y Wong, Y. (2013). Navigating corporate social responsibility components and strategic options: The IHR perspective. Academy of Strategic Management Journal, 12(1), 39-58. 